\title{
26 Research Square \\ Continuous Medical Education among resident physicians in Abha, Saudi Arabia: Current Practice and Existing Gaps
}

\section{Najwa Almoalwi}

Family medicine, $\mathrm{MOH}$

Safar Alsaleem ( $\square$ safar10abadi@hotmail.com )

King Khalid University https://orcid.org/0000-0002-2248-8620

Aesha Farheen Siddiqui.

King Khalid University

\section{Research article}

Keywords: Continuing Medical Education (CME), Residents, Abha

Posted Date: December 12th, 2019

DOI: https://doi.org/10.21203/rs.2.18686/v1

License: (c) (7) This work is licensed under a Creative Commons Attribution 4.0 International License. Read Full License 


\section{Abstract}

Background: Continuing Medical Education (CME) is concerned with the maintenance, improvement, and promotion of the health care and exhausts the physicians' working life-span. Although international research is extensive, only a dearth of studies exists in Saudi Arabia and the Middle East. This study aims to explore CME practices of resident physicians in Abha City and the existing gaps.

Method: A cross-sectional questionnaire-based descriptive study conducted on residents training in various healthcare facilities in Abha city.

Results: Participants in the study were $(n=300)$ doctors, of whom $(n=239,79.7 \%)$ reported attending lectures and seminars for their CME needs followed by $(n=119,39.7 \%)$ who attended case presentations. Electronic CMEs were used by a minority of $(n=24,8 \%)$, and only $(n=82,27.3 \%)$ attended journal clubs. Being busy was the main barrier against self-reading as per $(n=212,70.7 \%)$ of participants, and by $(n=155,51.7 \%)$ against lectures and seminars, and by $(n=124,41.3 \%)$ against courses attendance. There was no significant association between gender, nationality, or training level and satisfaction with CME activities $(p=0.982, p=0.924$, and $p=0.5400$ respectively). Satisfaction with CME activities varied considerably across specialties $(p=0.039)$, psychiatry trainees were far less satisfied than their restorative dentistry counterparts $(p=0.0046)$, as well as for General Surgery trainees $(P=0.0230)$, Ophthalmology trainees $(p=0.0301)$, pediatrics trainees $(p=0.0214)$, and Preventive Medicine trainees ( $p=0.0283$ ).

Discussion: The participant physicians favored contact CME activities but not non-contact CME activities. In consistence with global research findings, being busy was the main barrier against CME goals attainment. Participants affirmed that CME activities improved their clinical practice, clinical skills and academic skills.

Conclusion: Promotion of online learning in Saudi Arabia. Support of residents with protected time for their CME activities. Encouragement of trainees in to further advice about their preference in terms of CME forms to help boost their engagement

\section{Background}

Medical practitioners need to continuously update the knowledge in their specific fields to keep up with the proliferating medical knowledge and dynamic treatment technology [1]. Medical professionals continuing their training throughout their careers is termed Continuing Medical Education (CME). Formal CME includes organized activities such as conferences, workshops, symposia, courses, and educational meetings. Personal efforts such as frequent/habitual reading and inquiries that help a person to remain up to date in his/her professional development are also included [2].

Although formal CME may be a recent phenomenon, the concept of continuing medical education is not new. Health professionals have been involved in some form of CME since the early twentieth century [3]. Currently, continuing medical education (CME) is shifting from a traditional passive model to a competency-based, selfdirected learning model [4].

Continuing Medical Education (CME) is unending and lasts throughout the physician's working life. The primary purpose of continuing medical education is for professionals to remain up to date with the latest knowledge in their profession so as to facilitate competent practice and improve their patient care [4]. Continuing medical 
education helps to improve not only their knowledge and skills but also to build relationships that a physician may use to provide services for patients [5].

Without active learning, physicians are unable to remain competent for more than a few years after graduation. A review of recertification examination in the US reported that examinees who had been trained long before were less successful in the examination than those who were recently trained [6]. For physicians to stay fit to practice, it is thus a core professional responsibility if not an ethical and moral obligation to continue to learn throughout their career regardless of discipline, specialty, or type [7].

There are many issues facing health-related human resource development in the Kingdom of Saudi Arabia, and continuing medical education is not spared from these challenges. CME involves many actors such as providers, sponsors, participants, and regulators. Several challenges have been identified including a lack of transparency in the CME budget, which leads to a too close relationship between the pharmaceutical industry and physicians, as well as issues of coordination between providers, adequate assessment of needs and resources, effectiveness, and quality control [8]. In Saudi Arabia, the regulatory body that approves and accredits all CMEs, the Saudi Commission for Health Specialties (SCFHS), has ruled that all healthcare practitioners must acquire a set number of CME hours for successful licensing to practice in the Kingdom. Essentially, this regulation presumes that attending CME events secures the improvement of attendees' knowledge and skills [2]. However, this regulatory approach is not sufficient to ensure effective learning. There is a need to give proper attention to principles of adult learning to enable learners to be active participants in developing training programs. It is also necessary to fashion the learning activities according to learners' goals [9]. In Saudi Arabia, there have been no substantial reports on such aspects of CME [2]. Up to the researcher's knowledge, no similar study has been conducted in Abha City during the past decade to explore the needs and practices among resident physicians regarding their continuing education or to clarify trainees' views about different aspects of CME. This study aims to explore the CME practices of resident physicians in Abha City and to identify the barriers to continued education.

\section{Aim of Study}

This study aims to explore CME practices of resident physicians in Abha City and the existing gaps.

\section{Study Objectives}

1. To identify the current CME practices of resident physicians.

2. To explore opinions of resident physicians regarding continuing medical education.

3. To identify barriers in CME practice among resident physicians.

\section{Methods}

\section{Study design}

Cross-sectional correlational study.

\section{Setting of the study}


This cross-sectional analytical study involved all resident physicians at governmental care hospitals in Abha City (the Maternity and Children's Hospital and Aseer Central Hospital) 2018. The minimum sample size required for the present study was calculated to be 400 based on the following criteria: acceptable margin of error $5 \%$, confidence level $95 \%$, response distribution $50 \%$. A list of residents was obtained that yielded 400 currently enrolled residents from all departments. All residents were invited to participate in the study using an online questionnaire. A total of 313 residents returned a filled questionnaire for a response rate of $75 \%$. Thirteen incompletely filled questionnaires were discarded, leaving a final sample of 300.

\section{Data collection instrument}

This study used a self-administered questionnaire that was constructed by incorporating previous validated questionnaires obtained on request from their authors $[2,10]$. Besides the personal characteristics of age, gender, nationality, marital status, specialty, position, qualification, and years of experience, the various aspects of continuing professional education were explored. The aspects of CME that were included were as follows. Current participation in CME activities: frequency of CME activity, type of activity (conference, seminar, self-reading), reasons for doing $\mathrm{CME}$, satisfaction with current $\mathrm{CME}$ practices, preferred methods of instruction in different $\mathrm{CME}$ activities, and barriers to attending CME. Resident preferences for future CME activities (time of activity, duration, evaluation methods) were also elicited. We analyzed the total satisfaction score by adding up the ordinal responses of trainees according to the satisfaction score between 0 and 4 , where strongly disagree $=0$ and strongly agree $=4$. Cronbach's alpha for the 8 satisfaction questions was 0.85 , which indicates very good reliability and internal consistency. Obtaining all the necessary official approvals and research ethical committees prior to conduct the study.

\section{Data collection and analysis}

The study questionnaire was emailed to all residents working in the Asser region. Data were analyzed using the RStatistical Software version 3.4.1. Categorical data were summarized using frequencies and displayed using tables and bar graphs, while continuous data were summarized using median, range, mean, and standard deviation (SD). Both types of data are displayed in tables and graphs. Continuous data were approximately normally distributed. The effect of categorical and continuous variables on the outcome variable (satisfaction with $\mathrm{CME}$ ) was determined using linear regression, ANOVA, or a $t$-test as appropriate. The level of significance was set at $p \leq 0.05$.

\section{Results}

Total of 300 residents during year of 2018 academic year were participated in this study. Figure 1 presents the CME activity undertaken by the study participants in the past one year. During this period, 239 (79.7\%) of the participants reported attending lectures and seminars for their CME needs. There were 102 who attended workshops (34\%), whereas 89 (29.7\%) reported attending group discussions and 119 (39.7\%) reported attendance at case presentations. Electronic CMEs were used by a minority $(n=24,8 \%)$, and only $82(27.3 \%)$ attended journal clubs. There were 131 (43.7\%) who attended conferences. Note that residents usually engage in more than one modality of CME activities. 
Table 1 represents that there were 176 (58.7\%) males and 124 (41.3\%) females among the participating residents, most of whom were Saudi $(n=289,96.3 \%)$. Half of all residents were married. By specialty, $24.3 \%$ were residents in family medicine, $21.3 \%$ in internal medicine, and $12.7 \%$ in pediatrics. Other specialties, namely obstetrics and gynecology, ENT, and dermatology, made up approximately $5 \%$, and orthopedics, preventive medicine, and general surgery about $4 \%$ of study participants each. Lower figures were shown for ophthalmology (3.7\%), radiology (3.3\%), and psychiatry (2\%), while emergency medicine, restorative dentistry, and urology each made up less than $2 \%$ of the residents. By the level of residency, there were $89(29.7 \%)$ R1 residents; almost equal numbers of R2 and R3, 75 and 74 (25\% and $24.7 \%$ respectively); 44 (14.7\%) R4; and only 7 (2.3\%) were R5. 
Satisfaction with CME activities among the residents by characteristics

\begin{tabular}{|c|c|c|c|c|}
\hline Characteristic & $\begin{array}{l}\text { Frequency (n } \\
=300)\end{array}$ & $\begin{array}{l}\text { Percentage } \\
\text { (\%) }\end{array}$ & $\begin{array}{l}\text { Satisfaction score } \\
\text { Mean } \pm \text { SD }\end{array}$ & P-value \\
\hline $\begin{array}{l}\text { Gender } \\
\text { Males } \\
\text { Females }\end{array}$ & $\begin{array}{l}176 \\
124\end{array}$ & $\begin{array}{l}58.7 \% \\
41.3 \%\end{array}$ & $\begin{array}{l}21.017 \pm 5.310 \\
21.032 \pm 5.847\end{array}$ & 0.9816 \\
\hline $\begin{array}{l}\text { Age in years mean } \pm \text { standard deviation } \\
\text { (SD) (median) }\end{array}$ & $28.0 \pm 2.38$ & & $\begin{array}{l}\text { Estimate }=-0.079 \\
(0.134)\end{array}$ & 0.557 \\
\hline \multicolumn{5}{|l|}{ Marital Status } \\
\hline $\begin{array}{l}\text { Married } \\
\text { Single }\end{array}$ & $\begin{array}{l}151 \\
149\end{array}$ & $\begin{array}{l}50.3 \% \\
49.7 \%\end{array}$ & $\begin{array}{l}21.20 \pm 5.44 \\
20.85 \pm 5.65\end{array}$ & 0.5882 \\
\hline \multicolumn{5}{|l|}{ Nationality } \\
\hline Saudi & 289 & $96.3 \%$ & $21.017 \pm 5.541$ & 0.9236 \\
\hline Non-Saudi & 11 & $3.7 \%$ & $21.182 \pm 5.456$ & \\
\hline Training Specialty & & & & $\begin{array}{l}0.0393 \\
\text { (overall) }\end{array}$ \\
\hline Family Medicine & 73 & $24.3 \%$ & $21.88 \pm 5.19$ & 0.1403 \\
\hline Internal Medicine & 64 & $21.3 \%$ & $22.41 \pm 5.35$ & 0.2001 \\
\hline Pediatrics & 38 & $12.7 \%$ & $19.39 \pm 6.93$ & 0.0214 \\
\hline Obstetrics and Gynecology & 17 & $5.7 \%$ & $20.76 \pm 6.09$ & 0.0838 \\
\hline ENT & 15 & $5 \%$ & $20.07 \pm 4.30$ & 0.0531 \\
\hline Dermatology & 14 & $4.7 \%$ & $21.29 \pm 5.12$ & 0.1268 \\
\hline Orthopedics & 14 & $4.7 \%$ & $21.00 \pm 4.52$ & 0.1054 \\
\hline Preventative Medicine & 14 & $4.7 \%$ & $19.21 \pm 3.33$ & 0.0283 \\
\hline General Surgery & 12 & $4 \%$ & $18.83 \pm 6.71$ & 0.0230 \\
\hline Ophthalmology & 11 & $3.7 \%$ & $19.09 \pm 6.71$ & 0.0301 \\
\hline Radiology & 10 & $3.3 \%$ & $21.00 \pm 3.62$ & 0.1207 \\
\hline Psychiatry & 6 & $2 \%$ & $16.00 \pm 5.37$ & 0.0046 \\
\hline Emergency Medicine & 4 & $1.3 \%$ & $24.50 \pm 4.43$ & 0.6963 \\
\hline Restorative Dentistry & 4 & $1.3 \%$ & $26.00 \pm 1.83$ & \\
\hline Urology & 4 & $1.3 \%$ & $20.00 \pm 9.09$ & 0.1192 \\
\hline Experience years mean \pm SD (median) & $2.7 \pm 1.81$ & & & \\
\hline
\end{tabular}




\begin{tabular}{|lllll|}
\hline Characteristic & $\begin{array}{l}\text { Frequency } \\
\mathbf{9} \mathbf{( n 0 )}\end{array}$ & $\begin{array}{l}\text { Percentage } \\
\mathbf{( \% )}\end{array}$ & $\begin{array}{l}\text { Satisfaction score } \\
\text { Mean } \pm \text { SD }\end{array}$ & P-value \\
\hline Training Level & & & & \\
R1 & 89 & $29.7 \%$ & $20.27 \pm 4.85$ & 0.5400 \\
R2 & 75 & $25 \%$ & $21.19 \pm 6.72$ & overall \\
R3 & 74 & $24.7 \%$ & $21.47 \pm 5.14$ & Reference \\
R4 & 44 & $14.7 \%$ & $21.82 \pm 5.14$ & 0.2900 \\
R5 & 7 & $2.3 \%$ & $21.29 \pm 5.59$ & 0.1670 \\
& & & & 0.1290 \\
\hline
\end{tabular}

We analyzed the total satisfaction score by adding up the ordinal responses of residents by satisfaction score between 0 and 4 . A considerable number of residents rated the CME activities they received last year as good ( $\mathrm{n}=$ $101,33.7 \%)$ or very good $(n=26,8.7 \%)$, with $92(30.7 \%)$ thinking it was fair and $78(26 \%)$ believing it was poor. Only $3(1 \%)$ rated it as excellent (data not shown). There was no significant association between gender and satisfaction with CME activities $(p=0.982)$, and the mean satisfaction score with CME was slightly lower in male $(21.17 \pm 5.310)$ than female residents $(21.032 \pm 5.847)$. Also, we found no significant association between nationality and satisfaction with CME activities $(p=0.924)$; the mean satisfaction with CME score was almost same in non-Saudi residents (21.18 \pm 5.456$)$ and Saudi residents $(21.02 \pm 5.541)$. Satisfaction with CME activities was variable across specialties $(p=0.039)$, such that psychiatry residents were far less satisfied than their restorative dentistry counterparts $(p=0.0046)$, as well as for general surgery residents (mean $=18.83, p=0.0230)$, ophthalmology residents (mean $=19.09, p=0.0301$ ), pediatrics residents (mean $=19.39, p=0.0214$ ), and preventive medicine residents (mean $=19.21, p=0.0283$ ). The mean satisfaction with CME score for psychiatric residents was the lowest $(16.00 \pm 5.37)$, and the highest was for restorative dentistry residents $(26.00 \pm 1.83)$. There was no significant association between training level and satisfaction with CME activities $(p=0.54)$, and the mean satisfaction score with CME was slightly lower in R1 residents $(20.27 \pm 4.85)$ than R4 residents $(21.82 \pm$ 5.14). Similarly, age and years of experience did not show statistically significant associations with satisfaction score $(p=0.557$ and 0.673 , respectively)

Table 2 describes the residents' beliefs about continuous medical education as assessed by their level of agreement with various statements. To the statement, "I believe that my CME needs are currently satisfied," $42.3 \%$ of the residents agreed, compared to $24.3 \%$ who disagreed. To the statement, "I believe that my medical school education encouraged me to be an independent self-learner," $49.4 \%$ agreed and $24.3 \%$ were neutral. More than two-thirds (68.7\%) agreed that "I believe that CME activities should be organized on a national level." To the statement, "CME activities keep me up-to-date," a majority (65\%) agreed and 8\% disagreed. Some (68.7\%) agreed that "CME activities help me to improve my practice," in contrast to $8.3 \%$ who disagreed. A majority $(65 \%)$ agreed with the statement, "CME activities affect my professional confidence," compared to $26.7 \%$ who were neutral. As to the statement "CME activities offer new learning opportunities," $65.3 \%$ agreed and $7.7 \%$ disagreed or strongly disagreed, respectively. "CME activities provide sufficient scopes for questions and discussions" was agreed to by $57.7 \%$, while $10.7 \%$ disagreed. 
Table 2

Resident's beliefs about continuous medical education.

\begin{tabular}{|c|c|c|c|}
\hline Statement & Agree & Neutral & Disagree \\
\hline I believe that my CME needs are currently satisfied & $\begin{array}{l}127 \\
42.3 \%\end{array}$ & $\begin{array}{l}100 \\
33.3 \%\end{array}$ & $\begin{array}{l}73 \\
24.3 \%\end{array}$ \\
\hline $\begin{array}{l}\text { I believe that my medical school education encouraged me to be an } \\
\text { independent self-learner }\end{array}$ & $\begin{array}{l}148 \\
49.4 \%\end{array}$ & $\begin{array}{l}73 \\
24.3 \%\end{array}$ & $\begin{array}{l}79 \\
26.4 \%\end{array}$ \\
\hline I believe that CME activities should be organized on a national level & $\begin{array}{l}206 \\
68.7 \%\end{array}$ & $66,22 \%$ & $28,9.4 \%$ \\
\hline CME activities keep me up to date & $\begin{array}{l}195 \\
65 \%\end{array}$ & $81,27 \%$ & $24,8 \%$ \\
\hline CME activities help me to improve my practice & $\begin{array}{l}203 \\
68.7 \%\end{array}$ & $72,24 \%$ & $25,8.3 \%$ \\
\hline CME activities affect my professional confidence & $\begin{array}{l}195 \\
65 \%\end{array}$ & $\begin{array}{l}80 \\
26.7 \%\end{array}$ & $25,8.3 \%$ \\
\hline CME activities offer new learning opportunities & $\begin{array}{l}196 \\
65.3 \%\end{array}$ & $81,27 \%$ & $23,7.7 \%$ \\
\hline CME activities provide sufficient scope for questions and discussions & $\begin{array}{l}173 \\
57.7 \%\end{array}$ & $\begin{array}{l}95 \\
31.7 \%\end{array}$ & $\begin{array}{l}32 \\
10.7 \%\end{array}$ \\
\hline
\end{tabular}

Table 3 describes the residents' perceptions of the effectiveness of different CME activities. Regarding the effectiveness of CME activities, 141 (47\%) residents agreed that conferences and symposia "improved their clinical practice outcomes" and "improved their academic/teaching skills." Also, 184 (61.3\%) of participants agreed that workshops and courses "improved their clinical skills" and 171 (57\%) agreed that they also "improved their clinical practice outcomes." For inter-departmental activities, 164 (54.7\%) agreed that they "improved their academic/teaching skills" and 158 (52.7\%) agreed they "improved their clinical practice." 
Table 3

Residents' perceptions of the effectiveness of different CME activities.

\begin{tabular}{|c|c|c|c|c|c|c|c|c|c|c|}
\hline \multirow{5}{*}{$\begin{array}{l}\text { Benefit of CME } \\
\text { Activity }\end{array}$} & \multicolumn{10}{|c|}{ CME method analyzed for effectiveness } \\
\hline & \multirow{2}{*}{\multicolumn{3}{|c|}{$\begin{array}{l}\text { Conference/Symposia } \\
\mathrm{N}, \%\end{array}$}} & \multirow{3}{*}{\multicolumn{3}{|c|}{$\begin{array}{l}\text { Workshop/courses } \\
\text { N,\% }\end{array}$}} & \multirow{3}{*}{\multicolumn{3}{|c|}{$\begin{array}{l}\text { Inter-Departmental } \\
\text { Activities } \\
\text { N,\% }\end{array}$}} & \multirow[t]{4}{*}{$\mathbf{P}$} \\
\hline & & & & & & & & & & \\
\hline & & & & & & & & & & \\
\hline & A & B & C & A & B & C & A & B & C & \\
\hline $\begin{array}{l}\text { Retention of } \\
\text { knowledge }\end{array}$ & $\begin{array}{l}47 \\
15.7 \%\end{array}$ & $\begin{array}{l}144 \\
48 \%\end{array}$ & $\begin{array}{l}109 \\
36.3 \%\end{array}$ & $\begin{array}{l}37 \\
12.3 \%\end{array}$ & $\begin{array}{l}111 \\
37 \%\end{array}$ & $\begin{array}{l}152 \\
50.7 \%\end{array}$ & $\begin{array}{l}43 \\
14.3 \%\end{array}$ & $\begin{array}{l}124 \\
41.3 \%\end{array}$ & $\begin{array}{l}133 \\
44.3 \%\end{array}$ & 0.013 \\
\hline $\begin{array}{l}\text { Improving } \\
\text { attitude }\end{array}$ & $\begin{array}{l}55 \\
18.3 \%\end{array}$ & $\begin{array}{l}132 \\
44 \%\end{array}$ & $\begin{array}{l}113 \\
37.7 \%\end{array}$ & $\begin{array}{l}36 \\
12 \%\end{array}$ & $\begin{array}{l}117 \\
39 \%\end{array}$ & $\begin{array}{l}147 \\
49 \%\end{array}$ & $\begin{array}{l}40 \\
13.3 \%\end{array}$ & $\begin{array}{l}116 \\
38.7 \%\end{array}$ & $\begin{array}{l}144 \\
48 \%\end{array}$ & 0.025 \\
\hline $\begin{array}{l}\text { Improving } \\
\text { clinical skills }\end{array}$ & $\begin{array}{l}56 \\
18.7 \%\end{array}$ & $\begin{array}{l}127 \\
42.3 \%\end{array}$ & $\begin{array}{l}117 \\
39 \%\end{array}$ & $\begin{array}{l}21 \\
7 \%\end{array}$ & $\begin{array}{l}95 \\
31.7 \%\end{array}$ & $\begin{array}{l}184 \\
61.3 \%\end{array}$ & $\begin{array}{l}37 \\
12.3 \%\end{array}$ & $\begin{array}{l}108 \\
36 \%\end{array}$ & $\begin{array}{l}155 \\
51.7 \%\end{array}$ & $\begin{array}{l}< \\
0.001\end{array}$ \\
\hline $\begin{array}{l}\text { Improving } \\
\text { clinical } \\
\text { outcomes }\end{array}$ & $\begin{array}{l}42 \\
14 \%\end{array}$ & $\begin{array}{l}117 \\
39 \%\end{array}$ & $\begin{array}{l}141 \\
47 \%\end{array}$ & $\begin{array}{l}28 \\
9.3 \%\end{array}$ & $\begin{array}{l}\text { 101, } \\
33.7 \%\end{array}$ & $\begin{array}{l}171 \\
57 \%\end{array}$ & $\begin{array}{l}32 \\
10.7 \%\end{array}$ & $\begin{array}{l}110 \\
36.7 \%\end{array}$ & $\begin{array}{l}158 \\
52.7 \%\end{array}$ & 0.129 \\
\hline $\begin{array}{l}\text { Improving } \\
\text { managerial } \\
\text { skills }\end{array}$ & $\begin{array}{l}54 \\
18 \%\end{array}$ & $\begin{array}{l}131 \\
43.7 \%\end{array}$ & $\begin{array}{l}115 \\
38.3 \%\end{array}$ & $\begin{array}{l}29 \\
9.7 \%\end{array}$ & $\begin{array}{l}109 \\
36.3 \%\end{array}$ & $\begin{array}{l}162 \\
54 \%\end{array}$ & $\begin{array}{l}32, \\
10.7 \%\end{array}$ & $\begin{array}{l}122 \\
40.7 \%\end{array}$ & $\begin{array}{l}146 \\
48.7 \%\end{array}$ & $\begin{array}{l}< \\
0.001\end{array}$ \\
\hline $\begin{array}{l}\text { Improving } \\
\text { academic skills }\end{array}$ & $\begin{array}{l}56 \\
18.7 \%\end{array}$ & $\begin{array}{l}103 \\
34.3 \%\end{array}$ & $\begin{array}{l}141 \\
47 \%\end{array}$ & $\begin{array}{l}35 \\
11.7 \%\end{array}$ & $\begin{array}{l}101 \\
33.7 \%\end{array}$ & $\begin{array}{l}164 \\
53.3 \%\end{array}$ & $\begin{array}{l}30 \\
10 \%\end{array}$ & $\begin{array}{l}\text { 106, } \\
35.3 \%\end{array}$ & $\begin{array}{l}164 \\
54.7 \%\end{array}$ & 0.019 \\
\hline $\begin{array}{l}\text { Improving } \\
\text { communication } \\
\text { skills }\end{array}$ & $\begin{array}{l}54 \\
18 \%\end{array}$ & $\begin{array}{l}123 \\
41 \%\end{array}$ & $\begin{array}{l}127 \\
42.3 \%\end{array}$ & $\begin{array}{l}34 \\
11.3 \%\end{array}$ & $\begin{array}{l}106 \\
35.3 \%\end{array}$ & $\begin{array}{l}160 \\
53.3 \%\end{array}$ & $\begin{array}{l}42 \\
14 \%\end{array}$ & $\begin{array}{l}111 \\
37 \%\end{array}$ & $\begin{array}{l}147 \\
49 \%\end{array}$ & 0.055 \\
\hline $\begin{array}{l}\text { Improving } \\
\text { practice } \\
\text { behavior }\end{array}$ & $\begin{array}{l}50 \\
16.7 \%\end{array}$ & $\begin{array}{l}123 \\
41 \%\end{array}$ & $\begin{array}{l}127 \\
42.3 \%\end{array}$ & $\begin{array}{l}30 \\
10 \%\end{array}$ & $\begin{array}{l}100 \\
33.3 \%\end{array}$ & $\begin{array}{l}170 \\
56.7 \%\end{array}$ & $\begin{array}{l}39 \\
13 \%\end{array}$ & $\begin{array}{l}115 \\
38.3 \%\end{array}$ & $\begin{array}{l}146 \\
48.7 \%\end{array}$ & 0.008 \\
\hline $\begin{array}{l}\text { Improving } \\
\text { departmental } \\
\text { image }\end{array}$ & $\begin{array}{l}54 \\
18 \%\end{array}$ & $\begin{array}{l}131 \\
43.7 \%\end{array}$ & $\begin{array}{l}115 \\
38.3 \%\end{array}$ & $\begin{array}{l}40 \\
13.3 \%\end{array}$ & $\begin{array}{l}113, \\
37.7 \%\end{array}$ & $\begin{array}{l}147 \\
49 \%\end{array}$ & $\begin{array}{l}35 \\
11.7 \%\end{array}$ & $\begin{array}{l}111 \\
37 \%\end{array}$ & $\begin{array}{l}154 \\
51.3 \%\end{array}$ & 0.012 \\
\hline
\end{tabular}

Table 4 shows participants' preferred methods of instruction in the CME activities during the past one year. Most residents preferred that lectures should take the form of a conference/symposium $(n=111,37 \%)$. For demonstration-type CME activities, residents preferred workshops ( $n=119,39.7 \%)$, while for hands-on practice, most residents preferred workshops $(n=162,54 \%)$. Similarly, for small group CME practice, most residents preferred workshops $(n=108,36 \%)$. However, for live-case-presentation CMEs, residents equally preferred workshops and conferences ( $n=88,29.3 \% ; 89,29.7 \%$, respectively), while for simulation CMEs, workshops were the preference of $123(40.7 \%)$ of the residents. For distance learning CMEs and electronic meeting CMEs, a majority of residents preferred conferences $(n=119,39.7 \% ; n=134,44.7 \%$, respectively). 
Table 4

Residents' preferred methods of instruction in the CME activities during the past one year

\begin{tabular}{|lllll|}
\hline CME methods & $\begin{array}{l}\text { Conference } \\
\text { /Symposium }\end{array}$ & Workshop & Courses & Inter- Departmental Activities \\
\hline Lecturing & $111,37 \%$ & $1,0.3 \%$ & $60,20 \%$ & $77,25.7 \%$ \\
\hline Demonstration & $97,32.3 \%$ & $119,39.7 \%$ & $60,20 \%$ & $24,8 \%$ \\
\hline Hands-on practice & $50,16.7 \%$ & $162,54 \%$ & $49,16.3 \%$ & $39,13 \%$ \\
\hline Small group seminar & $53,17.7 \%$ & $108,36 \%$ & $68,22.7 \%$ & $72,24 \%$ \\
\hline Live case presentation & $89,29.7 \%$ & $88,29.3 \%$ & $50,16.7 \%$ & $74,24.7 \%$ \\
\hline Simulations & $57,19 \%$ & $123,40.7 \%$ & $55,18.3 \%$ & $66,22 \%$ \\
\hline Distant learning & $119,39.7 \%$ & $70,23.3 \%$ & $84,28 \%$ & $27,9 \%$ \\
\hline Electronic conferencing & $134,44.7 \%$ & $53,17.7 \%$ & $82,27.3 \%$ & $31,10.3 \%$ \\
\hline
\end{tabular}

Table 5 presents the frequency distribution of respondents by their preferred CME resource, frequency of CME activity, and reasons for using different CME activities and barriers. The most prevalent self-reading method was reading medical books $(n=230,76.7 \%)$, followed by online websites for self-reading $(n=196,65.3 \%)$. One in five residents reported reading medical journals, which was lower than the $30.3 \%$ who reported using social media for self-reading purposes. When asked how often they read, the majority $(n=128,42.7 \%)$ of residents reported weekly self-reading, compared to $116(38.7 \%)$ who reported daily self-reading. The reasons for using self-reading as a CME method were ease of time management $(n=208,69.3 \%)$, ease of place $(n=104,34.7 \%)$, price $(n=54,18 \%)$, subject $(n=86,28.7 \%)$, and reputation of provider $(n=13,4.3 \%)$. Barriers to self-reading were reported as being busy $(n=212,70.7 \%)$, lack of interest $(n=31,10.3 \%)$, lack of provision $(n=26,8.7 \%)$, lack of suitability $(n=15$, $5 \%)$, and high cost $(n=26,8.7 \%)$. 
Table 5

Distribution of residents by their preferred CME resource, frequency of CME activity, reasons to use different $\mathrm{CME}$ activities and barriers to attending $\mathrm{CME}$

\begin{tabular}{|c|c|c|c|}
\hline Variable & Self-Reading, n(\%) & Lectures, n(\%) & Courses n(\%) \\
\hline CME Resource & $\begin{array}{l}\text { Medical books } \\
\text { 230(76.7\%) } \\
\text { Medical journals } \\
63(21 \%) \\
\text { Online websites } \\
\text { 196(65.3\%) } \\
\text { Social media } \\
\text { 91(30.3\%) }\end{array}$ & $\begin{array}{l}\text { Conferences } \\
106(35.3 \%) \\
\text { Live casts } \\
74(24.7 \%) \\
\text { Presentations } \\
195(65.0 \%) \\
\text { Distance learning } \\
19(6.3 \%) \\
\text { Other methods } \\
13(4.3 \%)\end{array}$ & $\begin{array}{l}\text { Training courses } \\
186(62 \%) \\
\text { Workshops } \\
115(38.3 \%) \\
\text { Group discussions } \\
\text { 83(27.7\%) } \\
\text { Other } \\
16(5.3 \%)\end{array}$ \\
\hline $\begin{array}{l}\text { Frequency } \\
\text { weekly } \\
\text { daily } \\
\text { monthly } \\
\text { rarely }\end{array}$ & $\begin{array}{l}128(42.7 \%) \\
116(38.7 \%) \\
37(12.3 \%) \\
17(5.7 \%)\end{array}$ & $\begin{array}{l}130(43.3 \%) \\
46(15.3 \%) \\
88(29.3 \%) \\
57(19 \%)\end{array}$ & $\begin{array}{l}66(22 \%) \\
23(7.7 \%) \\
81(27 \%) \\
134(44.7 \%)\end{array}$ \\
\hline $\begin{array}{l}\text { Reason } \\
\text { time } \\
\text { place } \\
\text { price } \\
\text { subject } \\
\text { speaker certification } \\
\text { reputation of provider }\end{array}$ & $\begin{array}{l}208(69.3 \%) \\
104(34.7 \%) \\
54(18 \%) \\
86(28.7 \%) \\
13(4.3 \%)\end{array}$ & $\begin{array}{l}125(41.7 \%) \\
134(44.7 \%) \\
37(12.3 \%) \\
49(16.3 \%) \\
26(8.7 \%) \\
14(4.7 \%)\end{array}$ & $\begin{array}{l}122(40.7 \%) \\
112(37.3 \%) \\
39(13 \%) \\
73(24.3 \%) \\
36(12 \%) \\
23(7.7 \%)\end{array}$ \\
\hline $\begin{array}{l}\text { Barriers } \\
\text { being busy } \\
\text { lack of interest } \\
\text { lack of provision } \\
\text { lack of availability } \\
\text { lack of suitability } \\
\text { high cost }\end{array}$ & $\begin{array}{l}212(70.7 \%) \\
31(10.3 \%) \\
26(8.7 \%) \\
\text { NA } \\
15(5 \%) \\
26(8.7 \%)\end{array}$ & $\begin{array}{l}155(51.7 \%) \\
50(16.7 \%) \\
50(16.7) \\
67(22.3 \%) \\
18(6 \%) \\
37(12.3 \%)\end{array}$ & $\begin{array}{l}124(41.3 \%) \\
36(12 \%) \\
87(29 \%) \\
101(33.7 \%) \\
15(5 \%) \\
46(15.3 \%)\end{array}$ \\
\hline
\end{tabular}

Another method of CME was lectures and seminars, for which 106 (35.3\%) reported attendance at conferences, 74 (24.7\%) live casts, and 195 (65.0\%) presentations, and 19 (6.3\%) used distance learning and 13 (4.3\%) used other methods for lectures and seminars. In terms of the frequency of lectures and seminars for CME activities among residents, the majority $(n=130,43.3 \%)$ reported weekly attendance, compared to $46(15.3 \%)$ who reported daily use of lectures and seminars for CME needs. Also, 88 (29.3\%) reported monthly attendance, while 57 (19\%) reported rarely attending lectures or seminars. As for why they chose lectures and seminars as a CME method, time was reported by 125 (41.7\%), place by 134 (44.7\%), price by $37(12.3 \%)$, subject by 49 (16.3\%), speaker certification by $26(8.7 \%)$, and the reputation of the provider by $14(4.7 \%)$. Barriers to using lectures and seminars for CME activities as reported by residents were being busy by 155 (51.7\%), lack of interest by 50 (16.7\%), lack of provision by 50 (16.7\%), lack of availability by 67 (22.3\%), lack of suitability by 18 (6\%), and high cost by 37 (12.3\%).

Attending courses as a CME activity was reported by 186 (62\%) residents and 115 (38.3\%) residents reported attending workshops, while group discussions were reported by $83(27.7 \%)$ and $16(5.3 \%)$ used other methods for courses. In terms of the frequency of attending CME courses, the majority $(n=134,44.7 \%)$ reported rare attendance, compared to 81 (27\%) who reported monthly attending CME courses. However, 66 (22\%) reported 
weekly attendance and $23(7.7 \%)$ daily. In terms of the reason for choosing courses as a CME method, time was reported by $122(40.7 \%)$, place by $112(37.3 \%)$, price by $39(13 \%)$, subject by $73(24.3 \%)$, speaker certification by 36 $(12 \%)$, and reputation of provider by $23(7.7 \%)$. Barriers to courses were being busy $(n=124,41.3 \%)$, lack of interest $(n=36,12 \%)$, lack of provision $(n=87,29 \%)$, lack of availability $(n=101,33.7 \%)$, lack of suitability $(n=15$, $5 \%)$, and high cost $(n=46,15.3 \%)$.

The preferred duration for CME activities for the majority of participating residents ( $n=153,51 \%)$ was one to two days, followed by 137 (45.7\%) who preferred three to seven days. Only a minority preferred a month or three months $(n=2,0.7 \%)$ or less than a day or more than a week $(n=1,0.3 \%)$. The majority $(n=194,64.7 \%)$ preferred that CMEs should take place on a workday, compared to $106(35.3 \%)$ who preferred a non-workday. Mornings were preferred by most participants $(n=216,72 \%)$, with only $45(15 \%)$ preferring afternoons and $43(14.3 \%)$ evenings. Most participants $(n=167,55.7 \%)$ preferred that the duration of a CME activity should be limited to hours, 108 (36\%) preferred that it should be limited to days, and $26(8.7 \%)$ preferred a week limit for any CME activity. By method of evaluation, some $(n=135,45 \%)$ preferred a questionnaire evaluation for CME activities, compared to $114(38 \%)$ who preferred group discussion and $55(18.3 \%)$ who preferred verbal assessment.

\section{Discussion}

$\mathrm{CME}$, Continuing Medical Education, constitutes a wide range of educational activities that aim for the maintenance, development, and improvement of the knowledge, skills, and professional performance that enable practicing clinicians to provide safe and efficacious clinical services [11]. Lifelong learning remains an indispensable element in enhancing clinical knowledge and professional behavior among practicing clinicians [12], as clinical experience over the years does not necessarily yield higher levels of skills, professional behavior, or knowledge $[13,14]$. This study is unique in exploring various aspects of CME activities utilized by resident trainees in Abha City in Saudi Arabia, as well as their opinions of the effectiveness of CME activities and the barriers that hinder accessing them.

This study found that the most popular CME activity among the participating residents was lectures and seminars. Lectures are not just popular in our sample of trainees, but also remain the main source for CME. Around the world, studies exploring physicians' CME preferences agreed with our study findings $[5,15]$. Also, a recent exploratory study from Saudi Arabia confirmed that the majority of healthcare professionals preferred symposia and short courses [2]. Indeed, lectures are famous as one of the most common forms of dispensing knowledge among clinicians and have been found to have a positive impact on knowledge and skills, though they have very little performance-improving effect [5].The least popular CME activity was electronic activities. These are non-contact CME activities, which could be one of the reasons for their unpopularity among trainees [16].

Research has found that the more interaction there is between the adult learner and the educator, the greater the satisfaction with the learning method [15]. Electronic CME activities have certain sophisticated requirements, such as access to smart devices and software and the ability to afford extensive data connectivity costs [17]. Electronic CME activities have many advantages, such as flexibility and individualized learning format $[16,18]$, and trainees should be encouraged to make effective use of them. Notably, some studies did not find substantial differences between didactic lectures and online teaching methods in improving diagnostic skills among physicians $[19,20]$.

One of the main findings of this study is that the main barrier against attending all forms of CME was being busy, although it was higher for self-reading than contact CME like attending lectures and seminar courses. Our findings are a stark reminder of the reality of how busy clinical services are nowadays. No matter how motivated a clinician 
is, time pressures can hamper their CME goals attainment. This finding has been confirmed across national and international studies [21], and indeed little has changed in the past two decades, as a study in the same region some 20 years ago revealed that physicians had little time allocated for CME practice [22]. Time constraints were the second most common reason for not attending CME activities in a Pakistani sample of physicians [23]. The problem of a lack of protected time, we suspect, must have increased in recent times. Contrary to our findings, a recent study from the Eastern Region of Saudi Arabia found that lack of postgraduate education and dissatisfaction with CME activities were the main reported barriers to physicians' accessing CME activities [24]. Interestingly, that study found that high case load is an impetus for physicians to gain extra knowledge and skills and engage in CME more frequently [24].

Another reassuring finding in this paper that almost half of the participants affirmed that conferences improved their clinical practice and academic skills. Also, two out of every three residents confirmed that workshops and courses improved their clinical skills. This overall improvement in clinical skills as an effect of engaging in $\mathrm{CME}$ has been well established in several educational papers [25-28] and is consistent with the results of the current study. The results of our study are grounds for cautious optimism and point to improvements in physicians' satisfaction with CMEs over that reported in the last decade [10].

This study did not establish any background factors exerting a significant impact on satisfaction with CMEs, which agrees with the results of another study [24]. It is reassuring that gender was not a determining factor in satisfaction with CME activities. Only few differences were observed between training specialties, and it was worrying that psychiatric trainees were the least satisfied among the participants. Many surveys indicate that dental health professionals are in general more satisfied with CME activities than other health-care workers [29]. This could explain the differences in satisfaction scores between trainees in restorative dentistry and other medical trainees.

This study provided some much-needed answers. While it was comprehensive in assessing the CME practices and needs of the residents, it is limited by the fact that the study concerns resident physicians of one region and its results are not generalizable to all of KSA.

\section{Conclusion And Recommendations}

Based on the findings of this study, it is recommended that online learning be promoted as a CME format for trainees and practicing clinicians in Saudi Arabia. There should be support of residents and clinicians through provision of protected time for their CME activities outside their daily clinical commitments. Future research should focus on the effects of various forms of CME on clinical effectiveness as well as the professionalism and communication skills of physicians.

\section{Abbreviations}

Continuing Medical Education (CME), Number(n),Saudi Commission for Health Specialties (SCFHS),standard deviation (SD),Analysis of variance(ANOVA),statistical hypothesis test (t-test), The level of significance $(p)$,King Khalid university (KKU),Research ethical committee (REC),Ear nose throat (ENT),Residents first level(R1)

Residents second (R2), Residents third (R3), Residents fourth(R4), Residents fifth (R5). 


\section{Declarations}

\section{Acknowledgments:}

The authors would like to thank all the resident physicians, who despite their tight schedules agreed to participate in this seminal work. Without their contribution, this study would not have been possible. We also acknowledge the support of all those who facilitated this work.

\section{Authors' contributions}

NM was the main author of the manuscript. AF planned the study. NM and AF contributed to the data analysis and manuscript writing with assistant and supervision of SA. All authors approved submission of the final manuscript.

Funding: None

\section{Availability of data and materials:}

The data that support the findings of the current study are available from the corresponding author on reasonable request.

\section{Ethics approval and consent to participate:}

Informed consent was obtained from all residents prior to data collection. All the selected respondents were given assurance of confidentiality that the information gathered will be used exclusively for research purposes. This study was approved by the Institutional Review Board of the College of Medicine; King Khalid university (KKU) (Reference \#: REC: 2018/05/72).

All necessary official approvals to conduct this study were obtained.

Consent for publication: Not applicable.

Competing interests: The authors declare that they have no competing interests

\section{References}

1. Ahmed K, Ashrafian H. Life-long learning for physicians. Science. 2009;326(5950):227. doi:1126/science.326_227a. PMID19815754.

2. Alkhazim MA, Althubaiti A. Continuing medical education in Saudi Arabia: Experiences and perception of participants. J Health Spec. 2014;2:13-9.

3. Balmer JT. The transformation of continuing medical education (CME) in the United States. Adv Med Educ Pract. 2013;4:171-182. doi:10.2147/AMEP.S35087.

4. Moja L, Kwag KH. Point of care information services: a platform for self-directed continuing medical education for front line decision makers. Postgrad Med J.2015;91:83-91.

5. VanNieuwenborg L, Goossens M, De Lepeleire J, et al. Continuing medical education for general practitioners: a practice format. Postgrad Med J. 2016;92:217-22.

6. Buyske J. For the protection of the public and the good of the specialty: Maintenance of certification. Arch Surg. 2009;144(2):101-3. doi:10.1001/archsurg.2008.556 
7. Sehlbach C, Farr A, Allen M, et al. ERS Congress highlight: educational forum on continuing professional development. Breathe (Sheff). 2018;14(2):e12-e16. doi:10.1183/20734735.020918

8. Alghamdi AM. Challenges of continuing medical education in Saudi Arabia's hospitals. PhD dissertation, Newcastle University School of Medical Sciences Education Development, 2012. https://pdfs.semanticscholar.org/468d/8f512327af5ef98a74b04d641408ade75010.pdf

9. Davis DA, Prescott J, Fordis CM Jr, Greenberg SB, Dewey CM, Brigham T, et al. Rethinking CME: An imperative for academic medicine and faculty development. Acad Med. 2011;86:468-73.

10. Al-Mosilhi AH, Kurashi NY. Current situation of continuing medical education for primary health care physicians in Al-madinah Al-munawarah province, Saudi Arabia. J Fam Commun Med. 2006;13(2):75-82.

11. IASLC, International Association for the Study of Lung Cancer publications, accessed freely on 17 March 2019 at https://www.iaslc.org/sites/default/files/wysiwyg-assets/cme_definition_0.pdf

12. Veenstra GL, Ahaus K, Welker GA, et al. Rethinking clinical governance: healthcare professionals' views-a Delphi study. BMJ Open. 2017;7:e012591. doi: 10.1136/bmjopen-2016-012591

13. Bower EA. Education to return nonpracticing physicians to clinical activity: a case study in physician. J Contin Educ Health Prof. 2010;30(2):89-94.

14. Finlayson JR et al. Restoring professionalism: the physician fitness-for-duty evaluation. Gen Hosp Psychiatry. 2013;35(6):659-63.

15. Yee M, Simpson-Young V, Paton R, Zuo Y. How do GPs want to learn in the digital era? Aust Fam Physician. 2014;43(6):399-402.

16. Curran VR, Fleet LJ, Kirby F. A comparative evaluation of the effect of Internet-based CME delivery format on satisfaction, knowledge and confidence. BMC Med Educ. 2010;10:10. Published 2010 Jan 29. doi:10.1186/1472-6920-10-10

17. Hemmati N, Omrani S, Hemmati N. A comparison of internet-based learning and traditional classroom lecture to learn CPR for continuing medical education. Turk Online J Distance Educ. 2013;14:256-65.

18. Pullen D. Doctors online: learning using an internet based content management system. Int J Educ Dev Info Commun Technol. 2013;9:50-63.

19. McFadden P, Crim A. Comparison of the effectiveness of interactive didactic lecture versus online simulationbased CME programs directed at improving the diagnostic capabilities of primary care practitioners. J Contin Educ Health Prof. 2016;36(1):32-7.

20. Pugh CM, Arafat FO, Kwan C, Cohen ER, Kurashima Y, Vassiliou MC, Fried GM. Development and evaluation of a simulation-based continuing medical education course: beyond lectures and credit hours. Am J Surg. 2015;210(4):603-9.

21. Reed VA, Schifferdecker KE, Turco MG. Motivating learning and assessing outcomes in continuing medical education using a personal learning plan. J Contin Educ Health Prof. 2012;32:287-94.

22. Alsharif Al, Al-Khaldi YM. Attitude, practice and needs for continuing medical education among primary health care doctors in Asir region. J Family Community Med. 2001;8(3):37-44.

23. Ali SA, Hamiz ul Fawwad S, Ahmed G, Naz S, Waqar SA, Hareem A. Continuing medical education: A cross sectional study on a developing country's perspective. Sci Eng Ethics. 2018;24:251.

24. Alhejji A, Alramadan M, Aljasim M, Alramadhan B. Barriers to practicing continuous medical education among primary health care physicians in Alahsa, Kingdom of Saudi Arabia. J Health Edu Res Dev. 2015;3:147. 
25. Fils J, Bhashyam AR, Pierre Pierre JB, Meara JG, Dyer GS. Short-term performance improvement of a continuing medical education program in a low-income country. World J Surg. 2015;39(10):2407-12.

26. Fleet LJ, Fox G, Kirby F, Whitton C, Mclvor A. Evaluation outcomes resulting from an internet-based continuing professional development (CPD) asthma program: its impact on participants' knowledge and satisfaction. J Asthma. 2011;48(4):400-4.

27. Gist DL, Bhushan R, Hamarstrom E, Sluka P, Presta CM, Thompson JS, Kirsner RS. Impact of a performance improvement CME activity on the care and treatment of patients with psoriasis. J Am Acad Dermatol. 2015;72(3):516-23.

28. Vakani F, Jafri W, Bhulani N, Sheerani M, Jafri F. Physician satisfaction survey on continuing medical education. J Coll Physicians Surg Pak. 2012;22(1):69-70.

29. Bynum AB, Irwin CA, Cohen B. Satisfaction with a distance continuing education program for health professionals. Telemed J E Health. 2010;16(7):776-86

\section{Figures}

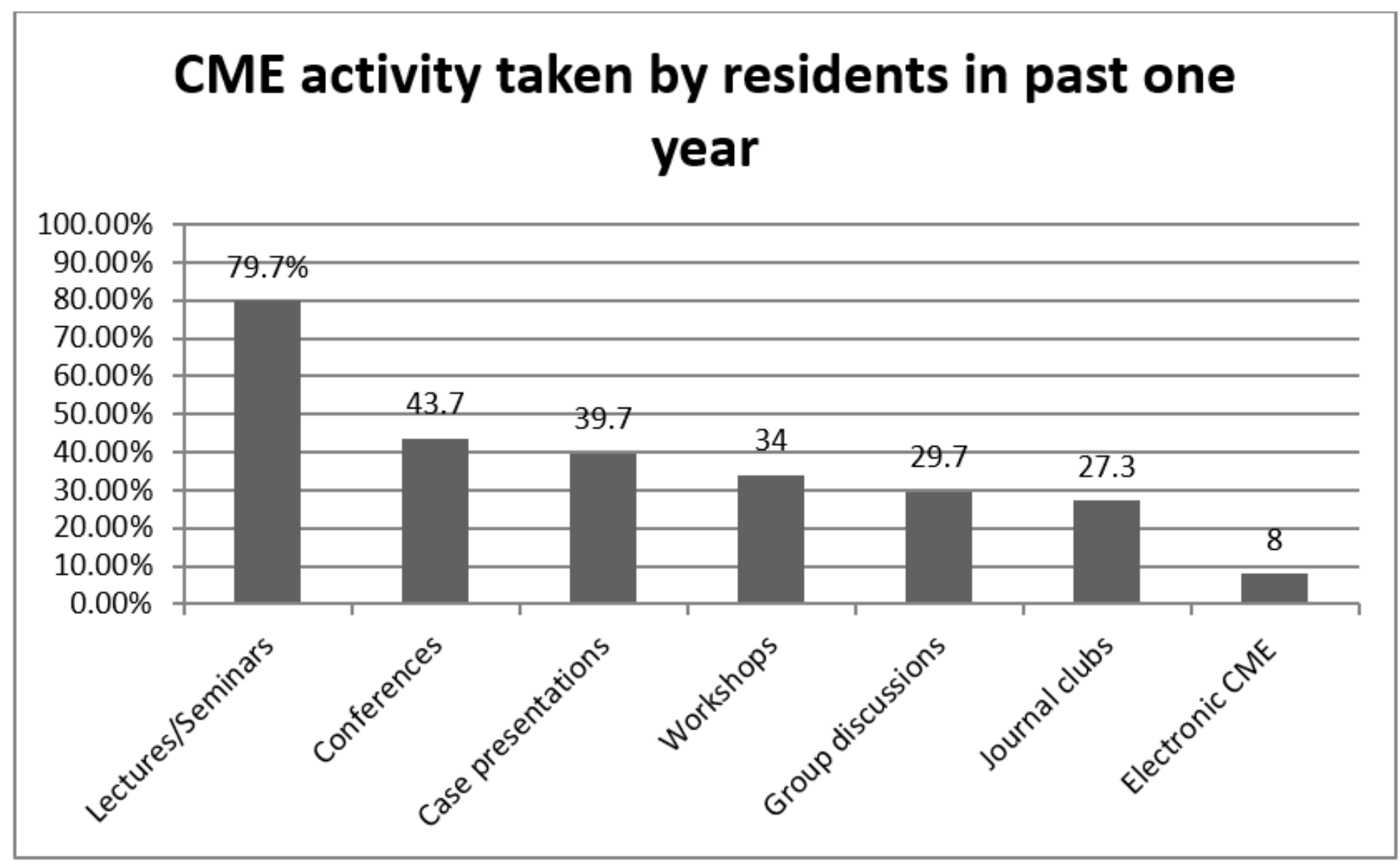

Figure 1

Distribution of CME activity taken by the residents in the past one year 


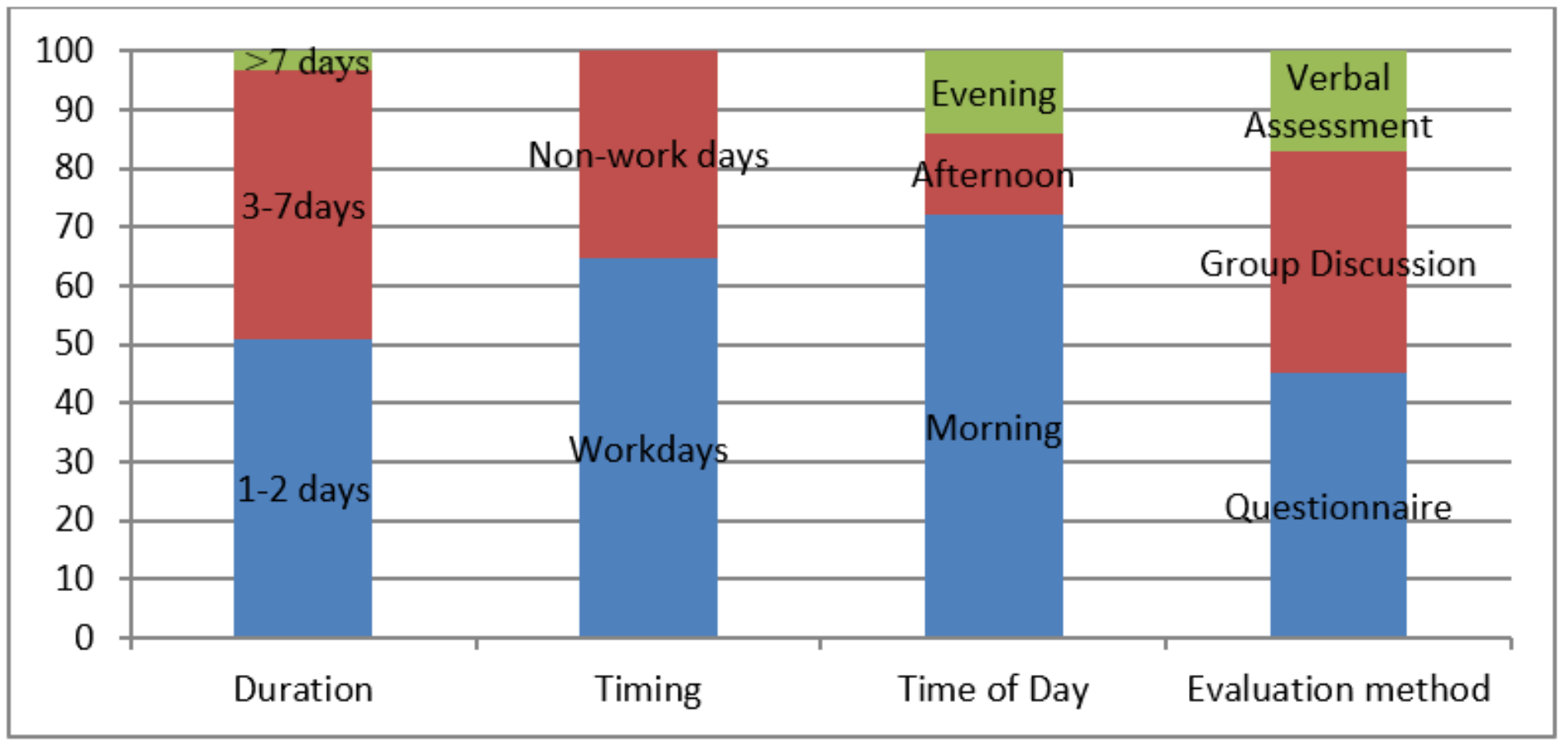

Figure 2

Residents' preferences regarding various aspects of CME activities

\section{Supplementary Files}

This is a list of supplementary files associated with this preprint. Click to download.

- ISSMSTROBEChecklistCME.pdf 